\title{
Gutzwiller wave function on a digital quantum computer
}

\author{
Bruno Murta $\oplus^{*}$ \\ QuantaLab, International Iberian Nanotechnology Laboratory (INL), 4715-330 Braga, Portugal \\ and Departamento de Física, Universidade do Minho, 4710-057 Braga, Portugal \\ J. Fernández-Rossier \\ QuantaLab, International Iberian Nanotechnology Laboratory (INL), 4715-330 Braga, Portugal \\ and Departamento de Física Aplicada, Universidad de Alicante, San Vicente del Raspeig 03690, Spain
}

(Received 30 March 2021; revised 11 June 2021; accepted 14 June 2021; published 21 June 2021)

\begin{abstract}
The determination of the ground state of quantum many-body systems via digital quantum computers rests upon the initialization of a sufficiently educated guess. This requirement becomes more stringent the greater the system. Preparing physically motivated Ansätze on quantum hardware is therefore important to achieve a quantum advantage in the simulation of correlated electrons. In this spirit, we introduce the Gutzwiller wave function (GWF) within the context of the digital quantum simulation of the Fermi-Hubbard model. We present a quantum routine to initialize the GWF that comprises two parts. In the first, the noninteracting state associated with the $U=0$ limit of the model is prepared. In the second, the nonunitary Gutzwiller operator that selectively removes states with doubly occupied sites from the wave function is performed by adding to every lattice site an ancilla qubit, the measurement of which in the $|0\rangle$ state confirms the operator was applied. Due to its nondeterministic nature, we estimate the success rate of the algorithm in generating the GWF as a function of the lattice size and the interaction strength $U / t$. The scaling of the quantum circuit metrics and its integration in general quantum simulation algorithms are also discussed.
\end{abstract}

DOI: 10.1103/PhysRevB.103.L241113

The quantum many-body problem permeates a wide range of fields of research within condensed matter physics, quantum chemistry, and materials science. In particular, it is the cornerstone of the electronic structure problem. Conventionalhardware-based numerical methods have played a pivotal role in unraveling the electronic structure of materials, but not without shortcomings. Indeed, although the low-energy properties of weakly interacting materials are well described by density-functional theory $[1,2]$ with approximate functionals based on the local density approximation [3], this approach often fails when strong electron-electron interactions prevail. Quantum Monte Carlo [4] methods are a leading alternative, though often plagued by the sign problem [5].

In principle, a more accurate description of correlated fermions could be achieved via wave-function-based methods, but the storage and manipulation of the wave function in classical hardware is hampered by the exponential wall problem [6]. This is, however, not the case in quantum hardware, owing to the principle of superposition and the natural encoding of entanglement. Quantum computers have thus been proposed [7] as a platform to simulate quantum many-body models that encapsulate the electronic structure of materials when their understanding demands an explicit representation of the wave function, either because of the presence of strong correlations or a high accuracy requirement [8].

\footnotetext{
*bpmurta@gmail.com
}

A number of quantum algorithms to determine the ground state of a given Hamiltonian $\mathcal{H}$ have been put forth. The most prominent example is quantum phase estimation [9] (QPE), whereby an initial state with a non-negligible overlap with the exact ground state undergoes a time evolution under the action of the propagator $e^{-i \mathcal{H} t / \hbar}$ subject to the control of ancilla qubits, from which the eigenspectrum can be extracted after the application of the inverse quantum Fourier transform. In particular, once the ground state energy is read out from the ancilla qubits, the state collapses into the exact ground state.

Because the resources required to implement QPE are far beyond the capacity of near-term quantum processors [10], a leaner class of hybrid variational algorithms [11] has been developed, of which the variational quantum eigensolver [12] (VQE) is the reference. In VQE, a parametrized state is prepared on a quantum computer, which is used to compute the expectation value of $\mathcal{H}$. This energy is then provided to a classical computer that performs the optimization routine to find the updated parameter values, which are then fed back to the quantum computer to begin the next iteration. Alternatively, the ground state can be found via quantum imaginary time evolution (QITE), which can be implemented in quantum hardware by casting it into a variational problem [13] or by finding the unitary operation [14] that transforms the state at the current step, $|\psi(\tau)\rangle$, into the (normalized) state at the next step, $|\psi(\tau+\Delta \tau)\rangle=\mathcal{N} e^{-\mathcal{H} \Delta \tau / \hbar}|\psi(\tau)\rangle$.

Despite the development of the aforementioned quantum simulation algorithms, it is well established [15] that the problem of finding the ground state of many Hamiltonians 
involving only local interactions is quantum Merlin-Arthur (QMA) complete [16]. Regarding QPE and QITE, the key challenge lies in the preparation of the initial state: The scaling of both algorithms is polynomial with respect to the inverse of the overlap between the initial state and the exact ground state [17], so the state preparation routine should lead to a polynomially decreasing overlap as the system size increases for the overall algorithm to be efficient [18]. Nevertheless, conventional choices of initial states, such as noninteracting or mean-field ground states, produce an exponentially vanishing probability of collapsing into the ground state due to the orthogonality catastrophe [19]. This overlap can in principle be enhanced via adiabatic evolution [20], but its success depends on the gap between the ground and first excited states throughout the adiabatic path, which is generally unknown.

Contrary to QPE and QITE, the challenge in VQE is not quite preparing the initial state, but rather formulating a parametrized Ansatz such that its manifold includes a path connecting the initial state to the exact ground state without incurring an exponential number of parameters. In any case, preparing an initial state with a greater overlap with the exact ground state could simplify the variational procedure by shortening the path in parameter space that needs to be covered, potentially reducing the number of optimization steps or the layers of the Ansatz. Perhaps even more important is the possible avoidance of barren plateaus [21], which can be linked to an uninformed initialization of the Ansatz [22].

Given the crucial role played by the initial state in the success of digital quantum simulation schemes, developing routines to prepare on quantum hardware physically motivated Ansätze is of great importance. It is within this context that, in this Letter, we propose an algorithm to prepare on quantum hardware the Gutzwiller wave function [23] (GWF). This is a conceptually simple, physically motivated Ansatz that contains a single parameter. It can be applied to the wide class of lattice models of correlated electrons where the electronelectron Coulomb repulsion is described, to leading order, in terms of the on-site Hubbard interaction [24] $U \hat{n}_{i, \uparrow} \hat{n}_{i, \downarrow}$, where $\hat{n}_{i, \alpha} \equiv \hat{a}_{i, \alpha}^{\dagger} \hat{a}_{i, \alpha}$ is the number operator acting on site $i$ for electrons of spin $\alpha=\uparrow, \downarrow$, with $\hat{a}_{i, \alpha}$ the annihilation operator in second quantization.

The Gutzwiller wave function is defined as

$$
\left|\psi_{G}\right\rangle=\prod_{i=1}^{N}\left(\mathbb{1}-g \hat{n}_{i, \uparrow} \hat{n}_{i, \downarrow}\right)\left|\psi_{0}\right\rangle,
$$

where $g \in[0,1]$ is a free parameter, $N$ is the number of lattice sites, and $\left|\psi_{0}\right\rangle$ is the noninteracting ground state. In words, the GWF is prepared by reducing the amplitude of the basis states of $\left|\psi_{0}\right\rangle$ with doubly occupied sites. The degree by which the amplitude is decreased is set by $g$. Although $g$ may, in principle, be allowed to vary from site to site in the absence of translational invariance, we shall assume the same $g$ is used for all sites to avoid having a number of free parameters scaling with $N$. In any case, for a sufficiently large system, the variation of $g$ across the lattice becomes negligible. The optimal value of $g$ for a given Hubbard parameter $U$ is found by minimizing the energy. The greater the magnitude of the on-site Hubbard interactions, the more unfavorable are the doubly occupied states, and hence the greater is $g$. In particular, when $U=0, g=0$, and when $U \rightarrow \infty, g=1$. Importantly, the Gutzwiller operator does not break any symmetry of the Hamiltonian.

In spite of its apparent simplicity, the GWF is a complex state that captures some correlations between the electrons. This complexity can be understood as follows. The noninteracting ground state

$$
\left|\psi_{0}\right\rangle=\prod_{\alpha, \sigma} \hat{a}_{\alpha, \sigma}^{\dagger}|0\rangle
$$

is a single Slater determinant, or a Fock state, when the single-particle basis $\{|\alpha\rangle\}$ is chosen to be the eigenbasis of the noninteracting tight-binding Hamiltonian. However, the Gutzwiller operator is applied in the site basis, therefore the operators $\left\{\hat{a}_{\alpha, \sigma}^{\dagger}\right\}$ have to be expanded as a linear combination of site operators $\left\{\hat{a}_{i, \sigma}^{\dagger}\right\}$,

$$
\left|\psi_{0}\right\rangle=\prod_{\alpha, \sigma}\left(\sum_{i} \phi_{\alpha}(i) \hat{a}_{i, \sigma}^{\dagger}\right)|0\rangle,
$$

in which case $\left|\psi_{0}\right\rangle$ appears as multideterminant state.

As a result, the determination of expectation values of the GWF is a many-body problem that cannot be solved exactly except in the special cases of one $[25,26]$ and infinite dimensions [27]. Hence, in two and three dimensions, numerical methods such as variational Monte Carlo $[28,29]$ have been employed to compute expectation values of the GWF. The GWF has been used to model a variety of correlated fermion problems, such as the metal-insulator transition [30], the lowtemperature behavior of ${ }^{3} \mathrm{He}$ [31], and the superconductivity in the cuprates [32].

In order to appreciate the significance of the GWF as an improved starting point for quantum simulation algorithms, we consider the Fermi-Hubbard model (FHM),

$$
\hat{\mathcal{H}}=-t \sum_{i=1}^{N} \sum_{\sigma=\uparrow, \downarrow}\left(\hat{a}_{i, \sigma}^{\dagger} \hat{a}_{i+1, \sigma}+\text { H.c. }\right)+U \sum_{i=1}^{N} \hat{n}_{i, \uparrow} \hat{n}_{i, \downarrow},
$$

at half filling in a one-dimensional chain with up to $N=12$ sites. Periodic boundary conditions could have been used just as well, but we chose to work with open boundary conditions to avoid the degeneracy of the noninteracting ground state when $N$ is a multiple of 4 . We compare the fidelity with respect to the exact ground state of three trial states: the GWF, the noninteracting, and the self-consistent mean-field ground states. The exact ground state is obtained via exact diagonalization using the numerical package QUSPIN [33]. The self-consistent mean-field theory amounts to a direct HartreeFock decoupling of the quartic Hubbard term, which gives rise to coefficients that depend on the expectation values of occupation numbers. Self-consistency is attained when singleparticle occupations of the mean-field ground state coincide (to a given precision) with the input values. Random initial conditions are used, and the lowest-energy state out of all trials is selected.

Figure 1(a) presents the fidelity of these three states with respect to the exact ground state for a chain of $N=12$ sites; similar profiles are observed for chains of different sizes. For concreteness, Fig. 1(b) shows how the fidelity of the three states decays with the size of the chain for the specific value of 

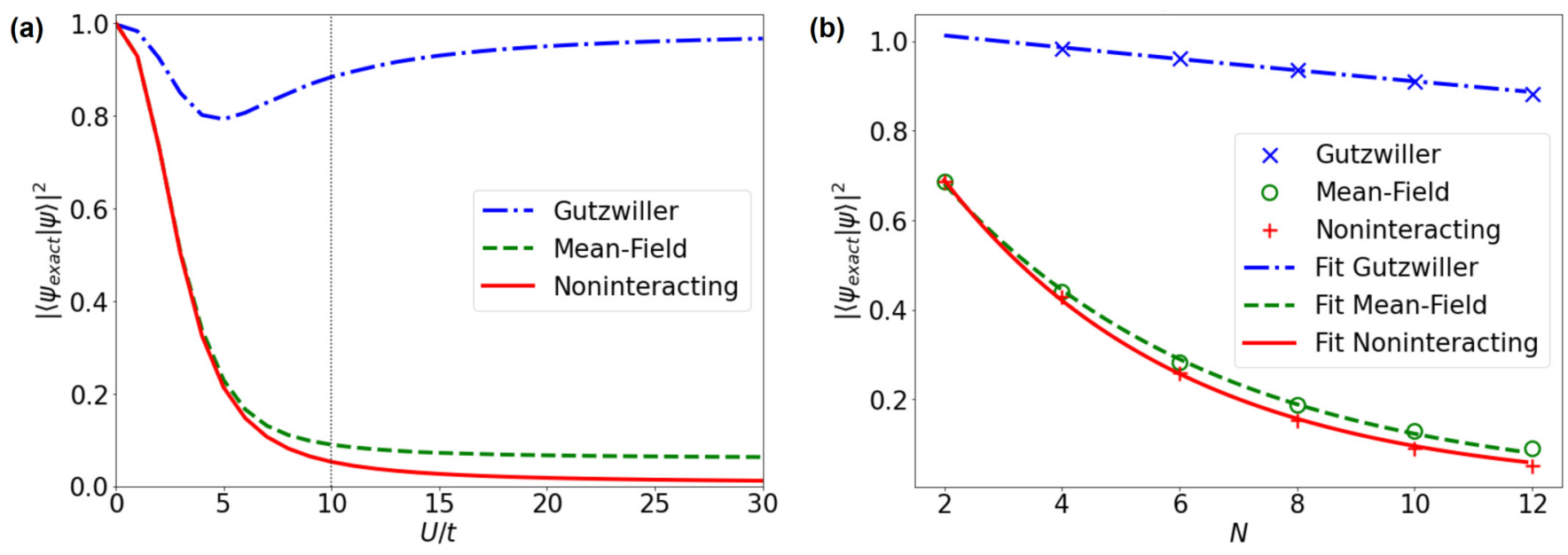

FIG. 1. Comparison of Gutzwiller wave function (GWF) to noninteracting and self-consistent mean-field ground states for the FermiHubbard model (FHM) in a chain with open boundary conditions at half filling. (a) Fidelity of three reference states with respect to the exact ground state for a chain of $N=12$ sites against a normalized Hubbard parameter $U / t$. (b) Same as (a), but now the size of chain $N$ is varied between 2 and 12 sites, while $U / t$ takes a fixed value of 10, as highlighted in (a) by the vertical dotted line. Numerical results were fitted to exponential decay $c_{1} e^{-c_{2} N}$.

$U / t=10$. The decrease of the overlap between the GWF and the exact ground state is significantly slower, thus rendering it a far better starting point for QPE, VQE, or QITE than the single-particle states, especially for large systems.

Henceforth, the initialization of the GWF on a digital quantum computer will be discussed. The Jordan-Wigner mapping [35] will be assumed, in which case the computational basis states $|0\rangle$ and $|1\rangle$ of each qubit encode the occupation of a spin orbital at a given site (unoccupied and occupied, respectively), yielding a total of $2 \mathrm{~N}$ qubits to represent the wave function, where the factor of 2 is due to the spin degeneracy.

Unsurprisingly, the algorithm proceeds in two stages, as shown in Fig. 2: First, the noninteracting ground state $\left|\psi_{0}\right\rangle$ is prepared, then the Gutzwiller operator is applied. For the preparation of $\left|\psi_{0}\right\rangle$, we follow previous works [36-38] based on the exploitation of the Thouless theorem $[39,40]$ and the expression of the resulting unitary operation as a quantum circuit using a $Q R$ decomposition [41] via Givens rotations [42]. As for the Gutzwiller operator, the challenge associated with its nonunitary character needs to be overcome. This can be accomplished by embedding the operator in a larger unitary operation, in a similar spirit to the implementation of the Jastrow operator [43].

Since the Gutzwiller operator acts on each site separately, let us consider a single site. Let us add an auxiliary qubit, to which the single-qubit gate

$$
\mathcal{U}(g)=\left(\begin{array}{cc}
1-g & -\sqrt{2 g-g^{2}} \\
\sqrt{2 g-g^{2}} & 1-g
\end{array}\right)
$$

is applied if and only if the two qubits that encode the occupations of the site are in state $|11\rangle$. Hence, for a given
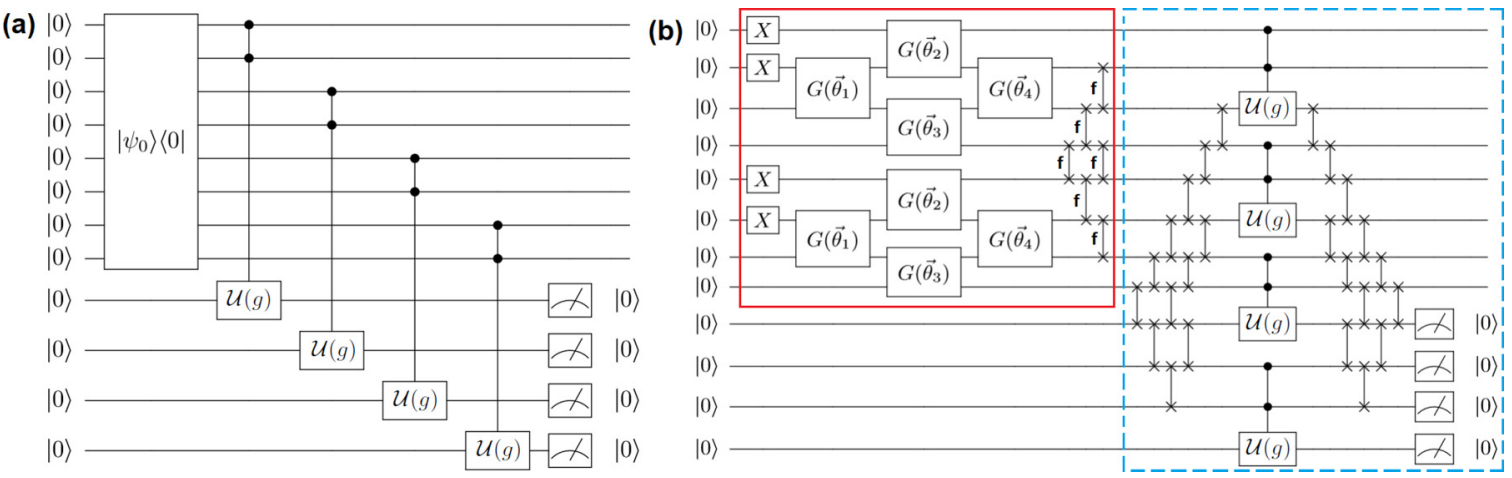

FIG. 2. (a) High-level scheme of routine to initialize GWF on a quantum computer, exemplified for a system of $N=4$ sites. After the noninteracting ground state $\left|\psi_{0}\right\rangle$ is prepared, a single-qubit rotation is applied to each auxiliary qubit if the two qubits that encode the occupations of the corresponding site are in state $|11\rangle$. Measuring the auxiliary qubits and retrieving only the trials that yield 0000 confirms the Gutzwiller operator was applied. (b) Detailed scheme of the routine to initialize GWF assuming linear qubit connectivity. The solid red box corresponds to the preparation of $\left|\psi_{0}\right\rangle$, which is first initialized in the diagonal basis and then transformed back to the original one via a Givens rotation decomposition, followed by the reordering of the qubits by site instead of spin, which involves fermionic SWAPs. The dashed blue box is the Gutzwiller operator: A network of SWAPs places auxiliary qubits next to the control qubits. A detailed description can be found in the Supplemental Material [34]. 
arbitrary two-qubit state $\quad|\Phi\rangle=c_{00}|00\rangle+c_{01}|01\rangle+$ $c_{10}|10\rangle+c_{11}|11\rangle$, the state after the $c c \mathcal{U}(g)$ reads

$$
\begin{aligned}
c c \mathcal{U}(|\Phi\rangle \otimes|0\rangle)= & \left(c_{00}|00\rangle+c_{01}|01\rangle+c_{10}|10\rangle\right) \otimes|0\rangle \\
& +c_{11}|11\rangle \otimes\left[(1-g)|0\rangle+\sqrt{2 g-g^{2}}|1\rangle\right] .
\end{aligned}
$$

Once the ancilla is measured in the computational basis, the state of the main register is collapsed into either $\left|\Phi_{0}\right\rangle=c_{00}|00\rangle+c_{10}|01\rangle+c_{10}|01\rangle+c_{11}(1-g)|11\rangle$ or $\left|\Phi_{1}\right\rangle=c_{11} \sqrt{2 g-g^{2}}|11\rangle$, where the subscript denotes the outcome of the readout. Notice that $\left|\Phi_{0}\right\rangle$ coincides with the action of the Gutzwiller operator on the initial state $|\Phi\rangle$. Hence, to guarantee that the Gutzwiller operator is applied to this site, the auxiliary qubit must be initialized in $|0\rangle$ and measured in $|0\rangle$ after the application of $c c \mathcal{U}(\mathrm{g})$. The method is thus nondeterministic.
The application of the Gutzwiller operator to the whole wave function merely amounts to repeating this procedure for every site, as illustrated in Fig. 2(a). For the sake of clarity, let us compute its action on the following four-site wave function explicitly:

$$
\begin{aligned}
|\psi\rangle= & a|\uparrow, \downarrow, \uparrow, \downarrow\rangle+b|\uparrow, \uparrow, \downarrow, \downarrow\rangle+c|\uparrow \downarrow, 0, \uparrow, \downarrow\rangle \\
& +d|\uparrow, \downarrow, \uparrow \downarrow, 0\rangle+e|\uparrow \downarrow, 0, \uparrow \downarrow, 0\rangle+f|\uparrow \downarrow, 0,0, \uparrow \downarrow\rangle .
\end{aligned}
$$

Applying the operator $\hat{P}_{G}(g) \equiv \prod_{i=1}^{N} \mathbb{1}-g \hat{n}_{i \uparrow} \hat{n}_{i \downarrow}$ gives

$$
\begin{aligned}
\hat{P}_{G}(g)|\psi\rangle= & a|\uparrow, \downarrow, \uparrow, \downarrow\rangle+b|\uparrow, \uparrow, \downarrow, \downarrow\rangle \\
& +(1-g)[c|\uparrow \downarrow, 0, \uparrow, \downarrow\rangle+d|\uparrow, \downarrow, \uparrow \downarrow, 0\rangle] \\
& +(1-g)^{2}[e|\uparrow \downarrow, 0, \uparrow \downarrow, 0\rangle+f|\uparrow \downarrow, 0,0, \uparrow \downarrow\rangle] .
\end{aligned}
$$

In words, the amplitude of the basis states with $n$ doubly occupied sites is reduced by a factor $(1-g)^{n}$. In turn, the action of the proposed routine on $|\psi\rangle$ is

$$
\begin{aligned}
& |0000\rangle \otimes[a|\uparrow, \downarrow, \uparrow, \downarrow\rangle+b|\uparrow, \uparrow, \downarrow, \downarrow\rangle+(1-g) c|\uparrow \downarrow, 0, \uparrow, \downarrow\rangle \\
& \left.\quad+(1-g) d|\uparrow, \downarrow, \uparrow \downarrow, 0\rangle+(1-g)^{2} e|\uparrow \downarrow, 0, \uparrow \downarrow, 0\rangle+(1-g)^{2} f|\uparrow \downarrow, 0,0, \uparrow \downarrow\rangle\right] \\
& \quad+|0001\rangle \otimes(1-g) \sqrt{2 g-g^{2}} f|\uparrow \downarrow, 0,0, \uparrow \downarrow\rangle+|0010\rangle \otimes\left[\sqrt{2 g-g^{2}} d|\uparrow, \downarrow, \uparrow \downarrow, 0\rangle+(1-g) \sqrt{2 g-g^{2}} e|\uparrow \downarrow, 0, \uparrow \downarrow, 0\rangle\right] \\
& \quad+|1000\rangle \otimes\left[\sqrt{2 g-g^{2}} c|\uparrow \downarrow, 0, \uparrow, \downarrow\rangle+(1-g) \sqrt{2 g-g^{2}} e|\uparrow \downarrow, 0, \uparrow \downarrow, 0\rangle+(1-g) \sqrt{2 g-g^{2}} f|\uparrow \downarrow, 0,0, \uparrow \downarrow\rangle\right] \\
& \quad+|1001\rangle \otimes\left(2 g-g^{2}\right) f|\uparrow \downarrow, 0,0, \uparrow \downarrow\rangle+|1010\rangle \otimes\left(2 g-g^{2}\right) e|\uparrow \downarrow, 0, \uparrow \downarrow, 0\rangle .
\end{aligned}
$$

The part of the wave function associated with the state $|0000\rangle$ in the auxiliary register coincides with $\hat{P}_{G}(g)|\psi\rangle$. Hence, all ancillas have to be measured in $|0\rangle$ to confirm the Gutzwiller operator was applied to the full state.

Given this method to apply the Gutzwiller operator on quantum hardware, the only piece left in the initialization of the GWF is the determination of the optimal value of $g$. This can be accomplished by minimizing the energy explicitly on the quantum computer, computing the expectation value of the Hamiltonian either via QPE, or by decomposing it in the Pauli basis, as in VQE. Nevertheless, one can deduce the optimal value of $g$ for a large system by extrapolating from small system simulations carried out on a conventional computer. This is indeed the case for the one-dimensional FHM at half filling, for which minor variations in the $g(U)$ relation are observed as the size of the chain varies. The same extrapolation should be possible for rectangular and square lattices. It should also be stressed that the quantum routine is applied identically in any number of spatial dimensions.

In the remainder of this Letter, we will discuss the scalability of the quantum routine herein proposed to initialize the GWF. In particular, the scaling of the relevant quantum circuit metrics [depth, width, and number of controlled-NOTs (CNOTs)] and of the number of repetitions due to the nondeterministic nature of the method will be detailed.

Regarding the implementation of the quantum circuit for the Gutzwiller operator, the resulting overhead is found to be analogous to that associated with the preparation of the noninteracting ground state. Given $N$ lattice sites, $N$ additional qubits are required to perform the Gutzwiller operator, so the circuit width is $3 N$ rather than $2 N$. As for the circuit depth, although the controlled rotations all act on independent trios of qubits, thereby allowing for their execution in parallel, the qubit connectivity must be taken into account, as the auxiliary qubits have to be placed in a position that is connected to the respective pair of control qubits [cf. Fig. 2(b)]. Making the realistic assumption of linear qubit connectivity [44], the Gutzwiller operator requires a total of $\mathcal{O}\left(6 N^{2}+18 N\right)$ CNOTs and a circuit depth [45] of $\mathcal{O}(12 N+12)$, which compares to the $\mathcal{O}\left(4 N^{2}-2 N\right)$ CNOTs (at half filling) and circuit depth of $\mathcal{O}(8 N-8)$ (at any filling) for the initialization of $\left|\psi_{0}\right\rangle$ [34]. It should be noted that, as shown in Fig. 2(b), in the initialization of $\left|\psi_{0}\right\rangle$ we include the reordering of the $2 N$ qubits by site instead of spin: The noninteracting FHM is spin polarized, so it is more practical to initialize $\left|\psi_{0}\right\rangle$ separately for each spin, but the propagator of the full Hamiltonian is more effectively implemented if the two qubits that represent the same site are next to each other $[46,47]$. In summary, the circuit depth, width, and number of CNOTs corresponding to the Gutzwiller operator are just a factor of $3 / 2$ greater than those for the preparation of $\left|\psi_{0}\right\rangle$. This analysis is independent of the dimensionality or the geometry of the lattice [34].

We now estimate the average number of times the quantum algorithm needs to be repeated for all $N$ ancillas to be found in the $|0\rangle$ state, thus ensuring a successful preparation of the GWF. This quantity depends both on $N$ and the value of $g$. In turn, $g$ depends on $U$. We have carried out this estimate numerically for chains with $N=2,4,6,8,10,12$ sites, and 
TABLE I. Average number of repetitions required to prepare GWF on a digital quantum computer for one-dimensional FHM at half filling with open boundary conditions. Values corresponding to $N=10$ sites were obtained directly from simulation, while the remaining ones were extrapolated from simulations of chains with $N=2,4,6,8,10,12$ sites. See Supplemental Material [34] for further details.

\begin{tabular}{lcccc}
\hline \hline & $N=10$ & $N=20$ & $N=30$ & $N=40$ \\
\hline$U / t=1$ & 2.7 & 6.5 & 16 & 39 \\
$U / t=5$ & 29 & 940 & 30700 & 1000000 \\
$U / t=10$ & 63 & 5900 & 550000 & 51000000 \\
$U / t=30$ & 77 & 9000 & 1000000 & 120000000 \\
$U / t=50$ & 78 & 9200 & 1100000 & 130000000 \\
\hline \hline
\end{tabular}

then extrapolated to larger chains. Table I shows the number of repetitions required to initialize the GWF for the FHM at half filling for multiple chain sizes. Even for as many as $N=$ 40 sites, the number of repetitions, though large, is clearly feasible for all $U / t$ values. We note that, in a similar spirit to $\mathrm{VQE}$, this Gutzwiller operator scheme corresponds to a relatively shallow circuit that must be repeated multiple times. However, whereas in VQE these repetitions serve to explore the parameter space in the search of the global minimum, in the Gutzwiller operator their cause is the nondeterministic nature of the routine.

However, if the GWF is needed as a starting point to carry out QPE or QITE, the improved fidelity does not offset the additional number of repetitions. This is illustrated in Table II, which compares the average number of repetitions due to the choice of the initial state that are required to find the exact ground state, taking the initial state to be the noninteracting ground state $\left(\left|\psi_{0}\right\rangle\right)$, the mean-field ground state $\left(\left|\psi_{\mathrm{MF}}\right\rangle\right)$, and the GWF, the latter case under two assumptions: excluding

TABLE II. Estimate of the average number of repetitions due to the choice of initial state $\left|\psi_{\text {initial }}\right\rangle$ that are required to find the exact ground state $\left|\psi_{\text {exact }}\right\rangle$ of a Fermi-Hubbard chain with $U / t=10$ at half filling via QPE or QITE. The initial states considered are the noninteracting ground state $\left(\left|\psi_{0}\right\rangle\right)$, the self-consistent mean-field ground state $\left(\left|\psi_{\mathrm{MF}}\right\rangle\right)$, and the GWF, the latter under two assumptions: excluding repetition overhead to initialize it $\left(\left|\psi_{G}\right\rangle^{*}\right)$, and including this overhead $\left(\left|\psi_{G}\right\rangle^{* *}\right)$. The number of trials due to the choice of initial state in QPE and QITE is estimated [34] as $1 /\left|\left\langle\psi_{\text {exact }} \mid \psi_{\text {initial }}\right\rangle\right|^{2}$. Values corresponding to $N=10$ sites were obtained directly from simulation, while the remaining ones were extrapolated from simulations of chains with $N=2,4,6,8,10,12$ sites.

\begin{tabular}{lcccc}
\hline \hline$N$ & 10 & 20 & 30 & 40 \\
\hline$\left|\psi_{0}\right\rangle$ & 11 & 120 & 1500 & 17000 \\
$\left|\psi_{\mathrm{MF}}\right\rangle$ & 7.8 & 70 & 580 & 5000 \\
$\left|\psi_{G}\right\rangle^{*}$ & 1.1 & 1.2 & 1.4 & 1.6 \\
$\left|\psi_{G}\right\rangle^{* *}$ & 69 & 7100 & 770000 & 82000000 \\
\hline \hline
\end{tabular}

the repetition overhead to prepare the $\operatorname{GWF}\left(\left|\psi_{G}\right\rangle^{*}\right)$, and including this overhead $\left(\left|\psi_{G}\right\rangle^{* *}\right)$. The repetition overhead overwhelms the savings arising from replacing $\left|\psi_{0}\right\rangle$ or $\left|\psi_{\mathrm{MF}}\right\rangle$ by $\left|\psi_{G}\right\rangle$. Likewise, the potential reduction of the number of layers or parameter updates in $\mathrm{VQE}$ arising from taking the GWF as the reference state will probably not compensate the increase in the number of repetitions, unless the barren plateau problem is especially acute.

One potentially promising route to tackle the repetition overhead issue is to combine this nondeterministic scheme with quantum amplitude amplification [48,49] for a suitable choice of oracle. This could lead to a trade-off between the number of repetitions and the circuit depth, thus making it a more viable solution for long-term quantum simulation algorithms such as QPE. A QITE-inspired approach, whereby the local nonunitary operator $\mathbb{1}-g \hat{n}_{i, \uparrow} \hat{n}_{i, \downarrow}$ is approximated by a unitary operation acting on a set of qubits that includes, but is not restricted to, the two qubits that encode site $i$, is also a viable option. Another line of research that could be especially relevant for near-term quantum simulation algorithms such as VQE is the preparation of the GWF via a tailored parametrized quantum circuit. The latter two methods have the additional benefit of avoiding the ancillas and controlled gates found in the current scheme.

Once the repetition overhead is overcome, further simulations of the Hubbard model with the GWF should be carried out, particularly for two-dimensional lattices, where a quantum advantage is likely to be attained first. It must be ensured that, for lattice sizes beyond the capacity of conventional numerical methods, the GWF suffices to achieve a high enough overlap with the exact ground state, otherwise more sophisticated methods that capture nonlocal correlations, such as Jastrow operators [43,50,51], may be required. Similar considerations hold for the application of the GWF to molecular systems, where the need to consider the full Coulomb interaction likely leads to a lower overlap with the exact ground state relative to what is observed for the Hubbard model.

In conclusion, the GWF was proposed as a promising trial state for the digital quantum simulation of strongly correlated electrons. We developed a routine to prepare the GWF on a gate-based quantum computer, the circuit depth and width requirements of which are similar to those associated with the initialization of the noninteracting ground state. The nondeterministic nature of this scheme leads to a repetition overhead that, though viable for the implementation of the GWF on its own, must be reduced in order to integrate it in general quantum simulation algorithms.

B.M. acknowledges support from the FCT Ph.D. Scholarship No. SFRH/BD/08444/2020. J.F.R. acknowledges financial support from the Spanish Government (Grant No. PID2019-109539GB-C41), and Generalitat Valenciana funding Prometeo 2017/139. The authors acknowledge use of the QUSPIN [33] package for the exact diagonalization calculations and of QISKIT [52] for the in silico simulations of the quantum circuits. 
[1] P. Hohenberg and W. Kohn, Phys. Rev. 136, B864 (1964).

[2] W. Kohn and L. J. Sham, Phys. Rev. 140, A1133 (1965).

[3] M. Lewin, E. Lieb, and R. Seiringer, Pure Appl. Anal. 2, 35 (2020).

[4] W. M. C. Foulkes, L. Mitas, R. J. Needs, and G. Rajagopal, Rev. Mod. Phys. 73, 33 (2001)

[5] M. Troyer and U.-J. Wiese, Phys. Rev. Lett. 94, 170201 (2005).

[6] W. Kohn, Rev. Mod. Phys. 71, 1253 (1999).

[7] R. P. Feynman, Int. J. Theor. Phys. 21, 467 (1982).

[8] Y. Cao, J. Romero, J. P. Olson, M. Degroote, P. D. Johnson, M. Kieferová, I. D. Kivlichan, T. Menke, B. Peropadre, N. P. Sawaya, S. Sim, L. Veis, and A. Aspuru-Guzik, Chem. Rev. 119, 10856 (2019).

[9] A. Y. Kitaev, arXiv:quant-ph/9511026.

[10] P. M. Q. Cruz, G. Catarina, R. Gautier, and J. FernándezRossier, Quantum Sci. Technol. 5, 044005 (2020).

[11] M. Cerezo, A. Arrasmith, R. Babbush, S. C. Benjamin, S. Endo, K. Fujii, J. R. McClean, K. Mitarai, X. Yuan, L. Cincio, and P. J. Coles, arXiv:2012.09265.

[12] A. Peruzzo, J. R. McClean, P. Shadbolt, M.-H. Yung, X.-Q. Zhou, P. J. Love, A.-G. Alán, and J. L. O’Brien, Nat. Commun. 5, 4213 (2014).

[13] S. McArdle, T. Jones, S. Endo, Y. Li, S. C. Benjamin, and X. Yuan, npj Quantum Inf. 5, 75 (2019).

[14] M. Motta, C. Sun, A. T. K. Tan, M. J. O’Rourke, E. Ye, A. J. Minnich, F. G. S. L. Brandão, and G. K.-L. Chan, Nat. Phys. 16, 205 (2020).

[15] J. Kempe, A. Kitaev, and O. Regev SIAM J. Comput. 35, 1070 (2006).

[16] QMA, short for quantum Merlin-Arthur, is the quantum analog of the nonprobabilistic complexity class NP.

[17] Y. Ge, J. Tura, and J. I. Cirac, J. Math. Phys. 60, 022202 (2019).

[18] S. McArdle, S. Endo, A. Aspuru-Guzik, S. C. Benjamin, and X. Yuan, Rev. Mod. Phys. 92, 015003 (2020).

[19] P. W. Anderson, Phys. Rev. Lett. 18, 1049 (1967).

[20] A. Aspuru-Guzik, A. D. Dutoi, P. J. Love, and M. Head-Gordon, Science 309, 1704 (2005).

[21] J. R. McClean, S. Boixo, V. N. Smelyanskiy, R. Babbush, and H. Neven, Nat. Commun. 9, 4812 (2018).

[22] Z. Holmes, K. Sharma, M. Cerezo, and P. J. Coles, arXiv:2101.02138.

[23] M. C. Gutzwiller, Phys. Rev. Lett. 10, 159 (1963).

[24] J. Hubbard, Proc. R. Soc. London, Ser. A 276, 238 (1963).

[25] W. Metzner and D. Vollhardt, Phys. Rev. Lett. 59, 121 (1987).

[26] F. Gebhard and D. Vollhardt, Phys. Rev. Lett. 59, 1472 (1987).

[27] M. C. Gutzwiller, Phys. Rev. 137, A1726 (1965).

[28] C. Gros, R. Joynt, and T. M. Rice, Phys. Rev. B 36, 381 (1987).

[29] H. Yokoyama and H. Shiba, J. Phys. Soc. Jpn. 56, 1490 (1987).

[30] W. F. Brinkman and T. M. Rice, Phys. Rev. B 2, 4302 (1970).

[31] D. Vollhardt, Rev. Mod. Phys. 56, 99 (1984).

[32] P. W. Anderson, Science 235, 1196 (1987).

[33] P. Weinberg and M. Bukov, QuSpin: a Python package for dynamics and exact diagonalization of quantum many-body systems, https://weinbe58.github.io/QuSpin/ (last accessed on March 23, 2021).
[34] See Supplemental Material at http://link.aps.org/supplemental/ 10.1103/PhysRevB.103.L241113 for a thorough account of the scaling of the relevant quantum circuit metrics (depth, width, and number of CNOTs) of the quantum routine proposed to implement the Gutzwiller projection, as well as a detailed explanation of the estimation of the number of repetitions required to initialize the Gutzwiller wave function and find the exact ground state via quantum phase estimation and imaginary time evolution.

[35] P. Jordan and E. Wigner, Z. Phys. 47, 631 (1928).

[36] D. Wecker, M. B. Hastings, N. Wiebe, B. K. Clark, C. Nayak, and M. Troyer, Phys. Rev. A 92, 062318 (2015).

[37] I. D. Kivlichan, J. McClean, N. Wiebe, C. Gidney, A. AspuruGuzik, G. K.-L. Chan, and R. Babbush, Phys. Rev. Lett. 120, 110501 (2018).

[38] Z. Jiang, K. J. Sung, K. Kechedzhi, V. N. Smelyanskiy, and S. Boixo, Phys. Rev. Appl. 9, 044036 (2018).

[39] D. J. Thouless, Nucl. Phys. 21, 225 (1960).

[40] R. Somma, G. Ortiz, J. E. Gubernatis, E. Knill, and R. Laflamme, Phys. Rev. A 65, 042323 (2002).

[41] R. A. Horn and C. R. Johnson, Matrix Analysis (Cambridge University Press, Cambridge, U.K., 1985).

[42] W. H. Press, S. A. Teukolsky, W. T. Vetterling, and B. P. Flannery, Numerical Recipes: The Art of Scientific Computing, 3rd ed. (Cambridge University Press, Cambridge, U.K., 2007).

[43] G. Mazzola, P. J. Ollitrault, P. K. Barkoutsos, and I. Tavernelli, Phys. Rev. Lett. 123, 130501 (2019).

[44] This assumption is particularly relevant for quantum computers based on superconducting circuits, for which the architectures typically only include linear connections for most qubits. As for trapped-ion quantum computers, there is, in principle, the possibility of achieving all-to-all connectivity, but, at the current state of development of the hardware, this comes at the cost of lower gate fidelities and greater execution times. Of course, if these technical limitations are overcome, trapped-ion quantum computers will allow us to implement this Gutzwiller operator routine with negligible circuit depth overhead.

[45] The estimation of the circuit depth only includes CNOT gates, since two-qubit gates have considerably greater execution times and error rates than single-qubit gates.

[46] Z. Cai, Phys. Rev. Appl. 14, 014059 (2020).

[47] C. Cade, L. Mineh, A. Montanaro, and S. Stanisic, Phys. Rev. B 102, 235122 (2020).

[48] S. J. Lomonaco and H. E. Brandt (eds.), in Quantum Computation and Information (American Mathematical Society, Providence, RI, 2002), Vol. 305.

[49] J. S. Van Dyke, G. S. Barron, N. J. Mayhall, E. Barnes, and S. E. Economou, arXiv:2103.13388.

[50] Y. Matsuzawa and Y. Kurashige, J. Chem. Theory Comput. 16, 944 (2020).

[51] F. Benfenati, G. Mazzola, C. Capecci, P. K. Barkoutsos, P. J. Ollitrault, I. Tavernelli, and L. Guidoni, arXiv:2101.09316.

[52] Qiskit: an open-source SDK for working with quantum computers at the level of pulses, circuits and application modules, https://qiskit.org/ (last accessed on March 23, 2021). 